\title{
Medium Altitude Long Endurance Unmanned Aerial Vehicles for Land Forces
}

\author{
Svajone BEKESIENE ${ }^{1}$, Leonas RUZGAS ${ }^{2}$ \\ 1,2 General Jonas Žemaitis Miltitary Academy of Lithuania, Silo str. 5A, LT-10322 Vilnius \\ E-mails: ${ }^{1}$ svajone.bekesiene@lka.lt
}

\begin{abstract}
Unmanned aerial vehicles (UAVs) have been developed for military purposes and are therefore particularly advanced and developed in military aviation. Under the circumstances, UAVs and drones are used these days not only in the military but also in the civilian industry.

Based on the chosen research objective and problems the questionnaire survey method was selected. There was collected primary data which was processed by software using a computer-based statistical analysis program.

Next analysis, which solve in part about goals and objectives was the experts' evaluation method based on experts' surveyed sample analysis. The Kendall's coefficient of concordance (W) was selected for experts' data analysis. The statistical software package SPSS version 26 was used for the collected experts' data analysis and for the hierarchical clustering analysis as well [11].
\end{abstract}

KEY WORDS: unmanned aircraft, unmanned aerial vehicle, Lithuanian Armed Forces, Land Forces

\section{Introduction}

The unmanned aerial vehicle (UAV) industry, which emerged in the mid-20th century, is still one of the most important direction of modern aviation. In the future, UAVs are expected to be able to replace many of the tasks performed by manned aircraft [1]. Unmanned aerial vehicles have been developed for military purposes and are therefore particularly progressive and advanced in military aviation. Under these circumstances, unmanned aircraft and drones are nowadays used not only in military but also in civilian industry.

The development of new technologies and their effective use in the military will provide advantage in future military conflicts $[2,10,12,15]$. The military has always been improving, and new technologies are needed to strengthen the army. Intelligence has always been highly valued and important in military conflicts, and has been collected in every possible way. The most commonly used method of reconnaissance is pedestrian reconnaissance. However, 21st Century technologies allow for much more efficient reconnaissance capabilities, like unmanned aerial vehicles with various types of sensors, which enable them to perform their intelligence tasks much faster and more efficiently.

Unmanned aircraft come in many types and sizes, are unmanned for their intended purpose, and are usually programmed to perform a specific task. Unmanned Aircraft is a prospect of future wars, as most missions will no longer have to endanger soldiers' lives. During the operation, the unmanned are capable of scanning the area with special equipment, providing real-time information, detecting enemy forces, marking them and, if necessary, destroying them. Unmanned troops can scan surrounding areas as enemy forces approach, protecting their own forces and carrying combat equipment. 57,000 pilots were killed in World War II, but today, most pilots' tasks can be performed by unmanned aerial vehicles [3].

The main advantage of UAVs is that they can perform tasks that are dangerous to the lives of soldiers and therefore can be used in extremely dangerous missions. Nowadays, reconnaissance and weaponry technologies of unmanned aerial vehicle are highly advanced, which is why they are used by the armies of most countries around the world. The medium altitude long endurance unmanned aerial vehicles with enhanced functionality can be used for the reconnaissance tasks, when patrol and unit guarding endanger the lives of soldiers.

As drone reconnaissance is the least life-threatening reconnaissance method, it was decided to describe the possibilities of using UAVs. Unmanned aerial vehicles are currently being developed and can be used for a variety of purposes, according to Reg Austin, author of "Unmanned Aircraft Systems", there are four main areas of unmanned aerial vehicle use [17]:

\footnotetext{
${ }^{1}$ Corresponding author.

E-mail address: svajone.bekesiene@1ka.lt
} 
- Reconnaissance;

- Operations;

- For retransmission of radio or other communication;

- Logistics.

The first and foremost function performed by UAVs is intelligence. UAVs begin to be used for intelligence purposes for many reasons, one of which is that a walking reconnaissance patrol fails to scout the area of operation or it takes a long time, resulting in - it is unknown whether the area is safe, in the long run. When the UAV rises to a certain height, it scans the area and sends the fixed data directly to the operator, thus speeding up the exploration process and increasing the accuracy of the information with the exact coordinates of the observed objects. In addition, there are supplementary means of reconnaissance, such as thermal imagers, night vision, infrared devices, that can be used for reconnaissance at any time of the day [17].

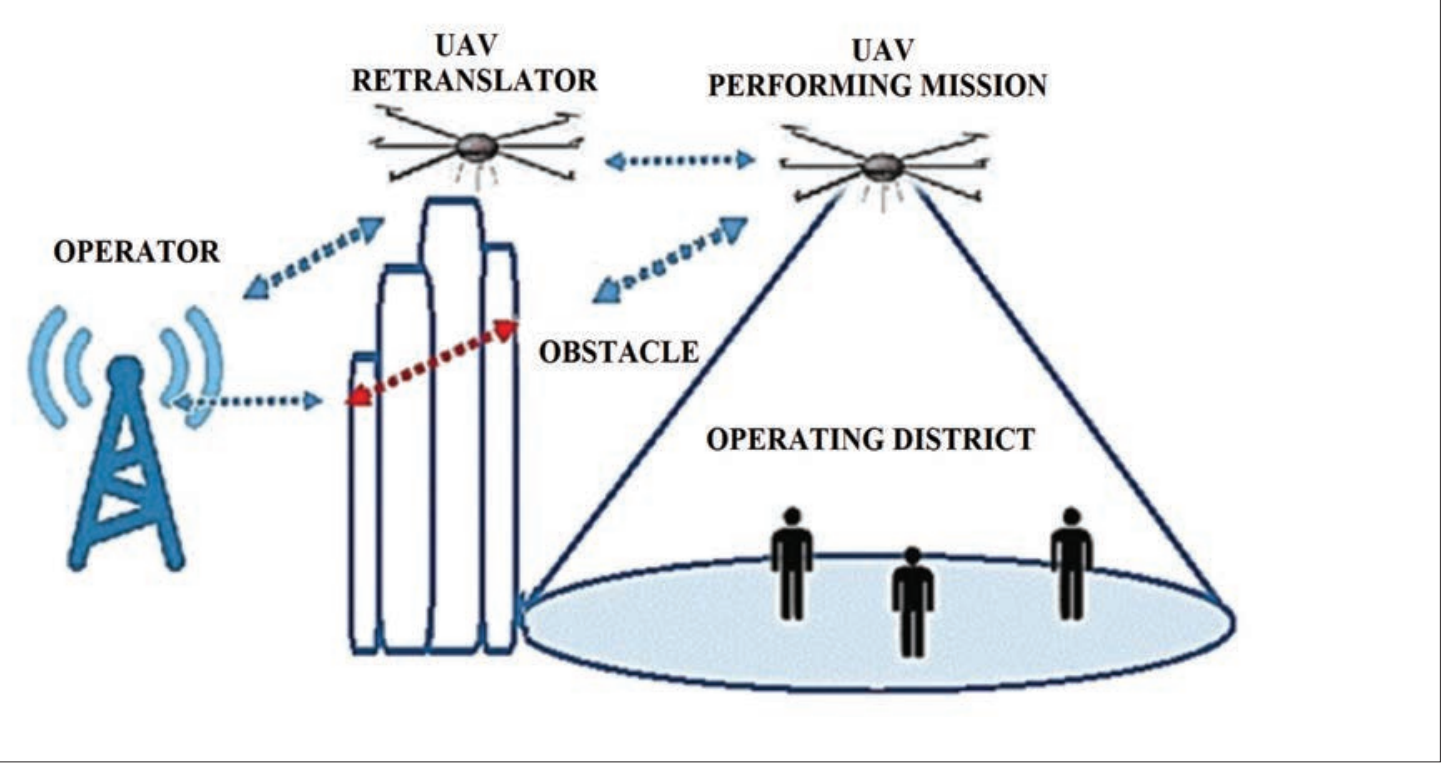

Fig. 1. Operating principle of unmanned aerial vehicles.

The second area of use is - operations. This function is divided into two directions: combat and reconnaissance operations. Reconnaissance operations are described above, so it remains to discuss combat. Tactical-class UAVs weighing up to $600 \mathrm{~kg}$, and others capable of carrying extra weight, in this case armament, which are used for combat operations. Combat drones have been in use for more than a decade, according to John David Bloom, and are therefore sophisticated and widely used by the world's major armies [20]. These days goal is to create a tactical category UAV capable of carrying light weapons.

A third use is to retransmit a radio or other communication to another UAV (see Figure 1). As is shown in the

Fig 1 the first UAV is closer to the operator, and transmits the signals sent from the operator to the further $\mathrm{UAV}$, thus increasing the range of operation of the UAV, which performing reconnaissance mission. In addition, this approach can be used when there is an obstacle between the mission-carrying UAV and the operator. This feature is a very good alternative to combat aircraft in the event of war, in the absence of a satellite link that controls large combat pilots such as the MQ-Reaper and others. In addition, this feature can be used to retransmit a radio communication between other radio stations when there is significant terrain interference.

The fourth function of UAV is logistics / supply. This type of UAV is most commonly used for delivery of supplies, and this function is also used in the public sector as a means of delivery of parcels [4]. This feature is very useful when your forces are surrounded and prevent all supply and communication routes by land. In this case, the UAV flies over its own forces and drops or lowers the cargo, such a method of supply delivery is safer and faster.

As was discussed above UAVs capabilities would provide an advantage during battle or operation, because the features offered by modern UAVs could support a variety of important tasks, such as: direct information retranslation of reconnaissance mission, combat operations or aerial support, radio retransmission in mountain areas, and surround supply. The aim of these investigations was to evaluate the application and use of medium-altitude long endurance unmanned aerial vehicles in the land forces. According to main goal, there was carried out the analysis of the functions and tasks of the Lithuanian Armed Forces, largest brigade. Additional there was conducted an analysis of the need for, and capabilities for, unmanned aerial vehicle enhanced brigade operations. 


\section{Methodology of Conducted Investigations}

These investigations were focused on the determination of the need to use the capabilities of medium-height enhanced functionality drones in the Lithuanian Army Land Forces (LALF). The method of qualitative research was chosen to implement the tasks of the work and achieve the main goal. In order to determine the appropriate types of LALF drones and their deployment possibilities, the following empirical research methods were used:

- Expert survey (questionnaire survey), the qualitative research method was used to obtain the information necessary to perform the research tasks, in this case, to determine the need to use medium-altitude unmanned aerial vehicles.

- Case study, the qualitative research method with the aim to identify a more efficient course of action and to compare the need with the available capacity, in this case, the improvement of the main tasks of MPB GV with UAVs.

Chosen research methods helped to identify the need for the use of enhanced functionality drones. Specific questions were used to questionnaire the UAVs' operators who were accepted as the experts. The results of the survey were systematized and summarized. Moreover, the level of agreement of the experts' opinions was assessed according to the concordance coefficient.

\subsection{Design of the Research}

Taking into account qualitative study requirements focus group data collection method as most suitable for these multiple perspectives obtaining regarding the same problem was used. Focus group discussions helped to show visibly different perspectives for problem-solving, offer observable interactions and capture the experiences and views with specific issues closely related to UAVs research questions.

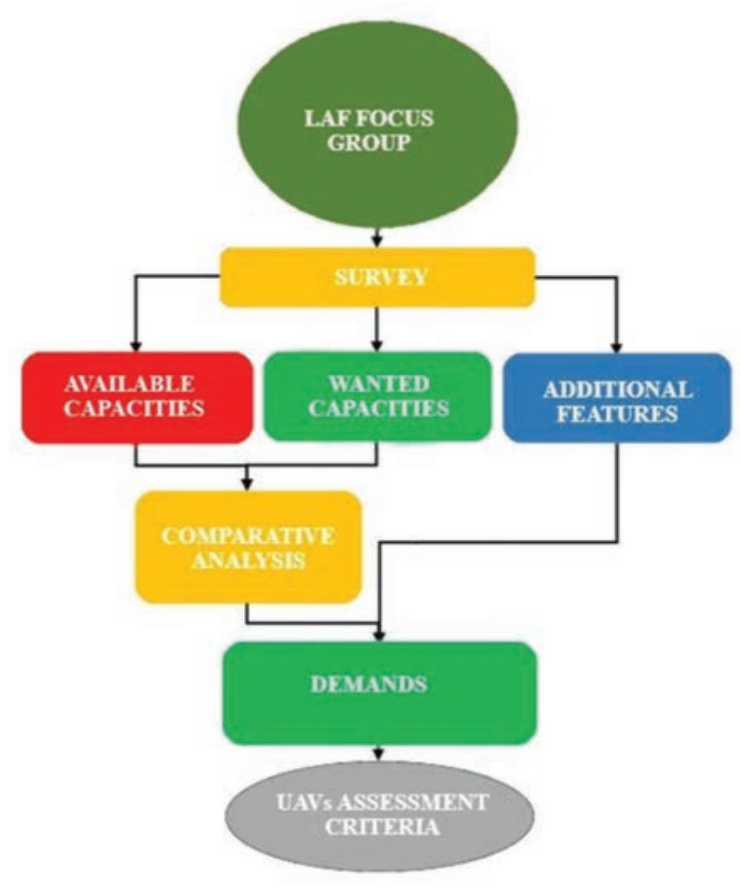

Fig. 2. Conducted study design.

Three categories of information were obtained from the focused group of experts: about the available capacities, the need for capacities, and additional functions. The questionnaire consisted of 20 questions, using a threestage system. First stage: 7 questions to find out the available capacity. Phase 2: 7 questions to identify capacity needs. Step Three: 4 questions to find out the need for additional features. Comparing the available and required capacities, the difference between the required capacities was obtained and the need was obtained by adding additional functions (see Figure 2). 


\subsection{The Selection of Experts}

The respondents as experts were selected according to the main criteria - experience and work with drones. Lithuanian Special Operations Force has been using drones since 2017, the experience is not very big, so it was decided to additionally interview the LAF UAVs specialists about additional functions. Two focused groups of respondents participate in the survey. The first focus group (6 respondents) - UAVs operators from the Lithuanian Grand Duke Algirdas mechanized infantry, Grand Duchess Birute Ulonai, King Mindaugas hussars, and Duke Vaidotas mechanized infantry, battalions. The reconnaissance units of these battalions use the UAVs to perform their assigned tasks, so it was chosen to interview at least one reconnaissance specialist from each battalion. The second focus group of respondents was a group of specialists of the LASCO Air Force working with drones (3 respondents).

Determining the number of experts was based on methodological assumptions formulated in test theory, which states that the reliability of aggregated decisions and the number of decision makers (in this case operators) are related by a rapidly fading nonlinear relationship. It has been proved that in modules of aggregated expert assessments with equal weights, the accuracy of decisions and assessments of a small group of experts does not inferior to the accuracy of decisions and assessments of a large group of experts, therefore a focused group was chosen.

The study questionnaire was compiled according to the methodological instructions. The questionnaire contains closed alternative questions with answer options (the respondent is allowed to choose one or more answer options depending on the question). Another type of questions used in the questionnaire is open-ended questions (the respondent is allowed to express his / her opinion).

Study procedure - the first study is divided into two stages. In the first stage, 6 experts from MPB GV were interviewed, in the second stage, 3 Air Force specialists working with UAVs were interviewed. The purpose of the questionnaire is to identify the available and required capacities and characteristics and to review the opinion of specialists on the need for additional functions. The results of the survey are compared and systematized with the aim to find out the need for specific tasks according to the specifics of the departments.

\subsection{The Concordance Coefficient Calculation Methodology}

Expert assessments are used in many studies. Expert assessment requires special expert knowledge and expertise, in this case the operation and use of drones, which are available to only a small number of specialists serving in the LASCO, therefore the focus group experience assessment method was chosen. Based on expert responses, a study on the need for enhanced functionality drones was conducted. An expert individual assessment method was chosen for the research - a questionnaire survey.

The Kendall's coefficient of concordance (W) was selected for collected experts' data analysis. This coefficient usually is used to prove the agreement among several (p) experts who are assessing a given set of $\mathrm{n}$ objects. For data analysis was used SPSS 26v [11].

From mathematical point of view there are two possible calculations to found Kendall's W statistic, but the result will be reached the same. $\mathrm{S}$ or $\mathrm{S}$ ' is calculated first from the row-marginal sums of ranks Ri received by the objects:

or

$$
S=\sum_{i=1}^{n}\left(R_{i}-\bar{R}\right)^{2}
$$

$$
S^{\prime}=\sum_{i=1}^{n} R_{i}^{2}=S S R .
$$

Where :

$\mathrm{S}$ is a sum-of-squares statistic over the row sums of ranks;

$R_{i}$ row sums of ranks;

$R$ is the mean of the $R_{i}$ values.

Kendall's W statistic can be obtained from one of the following formulas:

or

$$
\begin{aligned}
& W=\frac{12 S}{p^{2}\left(n^{3}-n\right)-p T} \\
& W=\frac{12 S^{\prime}-3 p^{2} n(n+1)^{2}}{p^{2}\left(n^{3}-n\right)-p T},
\end{aligned}
$$


where

$\mathrm{n}$ is the number of objects,

$\mathrm{p}$ the number of judges.

$\mathrm{T}$ is a correction factor for tied ranks [16].

Kendall's W statistic is an estimate of the variance of the row sums of ranks $R_{i}$ divided by the maximum possible value the variance can take; this occurs when all judges are in total agreement; hence $0 \leq \mathrm{W} \leq 1$. To derive the formulas for $\mathrm{W}$ given above, one has to know that the sum of all ranks in the data table is $p n(n+1) / 2$ and that the sum of squares of all ranks is $p^{2} n(n+1)(2 n+1) / 6$.

SPSS 26v. package allows us to verify the null hypothesis of equality of concordance coefficient to zero. Concordance statistically significant if the $\mathrm{p}$ - value is less than the selected significance level $\alpha$ (it can be found in the column as "Asymp. Sig.").

All mathematical operations were performed on collected data. The investigation results are presented in the section 3 .

\section{Investigation Results and Discussion}

After summarizing the LLF respondents' responses the radar diagrams of the available capacities were created. Schematically represented the characteristics of the available capacities of LLF SOP can be seen in Fig. 3, and the available capacities of LLF Air Forces can be seen in Fig. 4. The systematized functions of available capacity are plotted on a scale of 1 to 5, depending on the experts' answers.

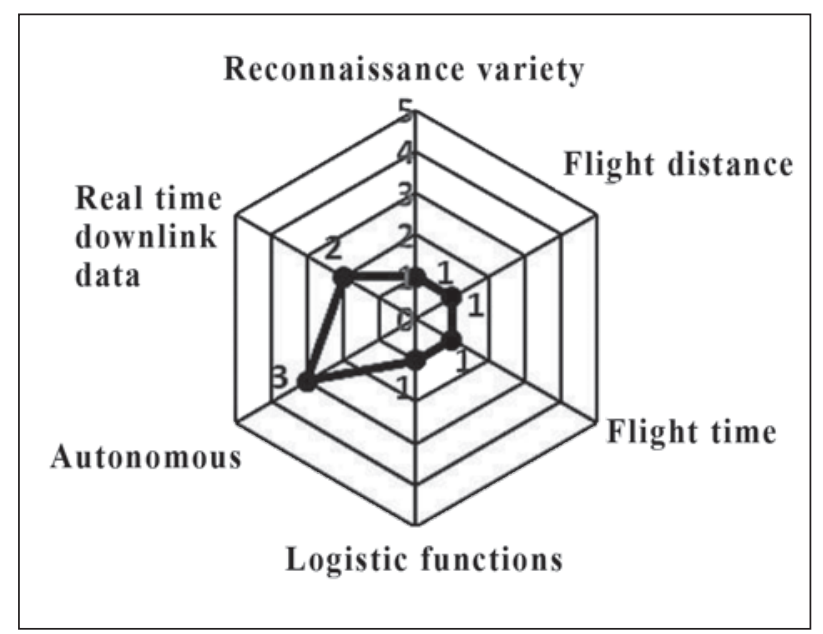

Fig.3. The available capacities of LLF SOP. Source: authors' research result

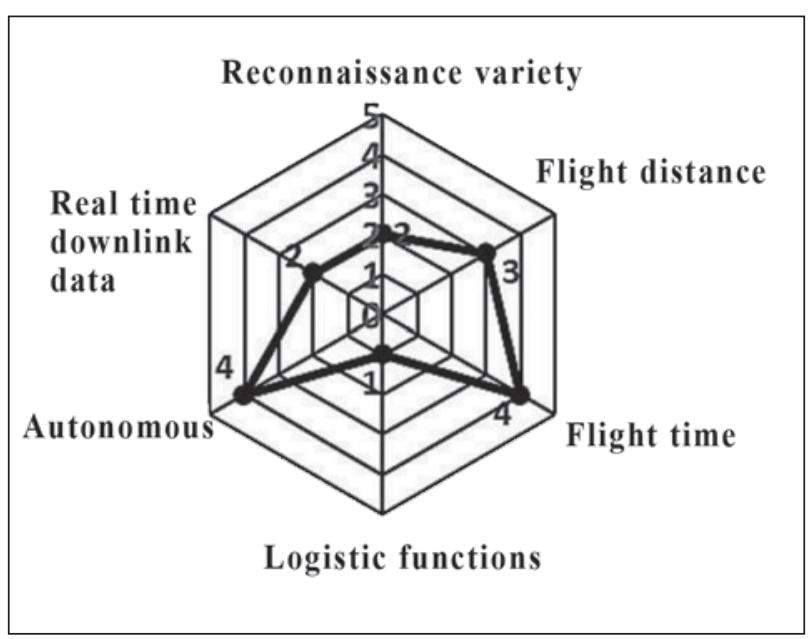

Fig. 4. The available capacities of LLF Air Forces. Source: authors' research result

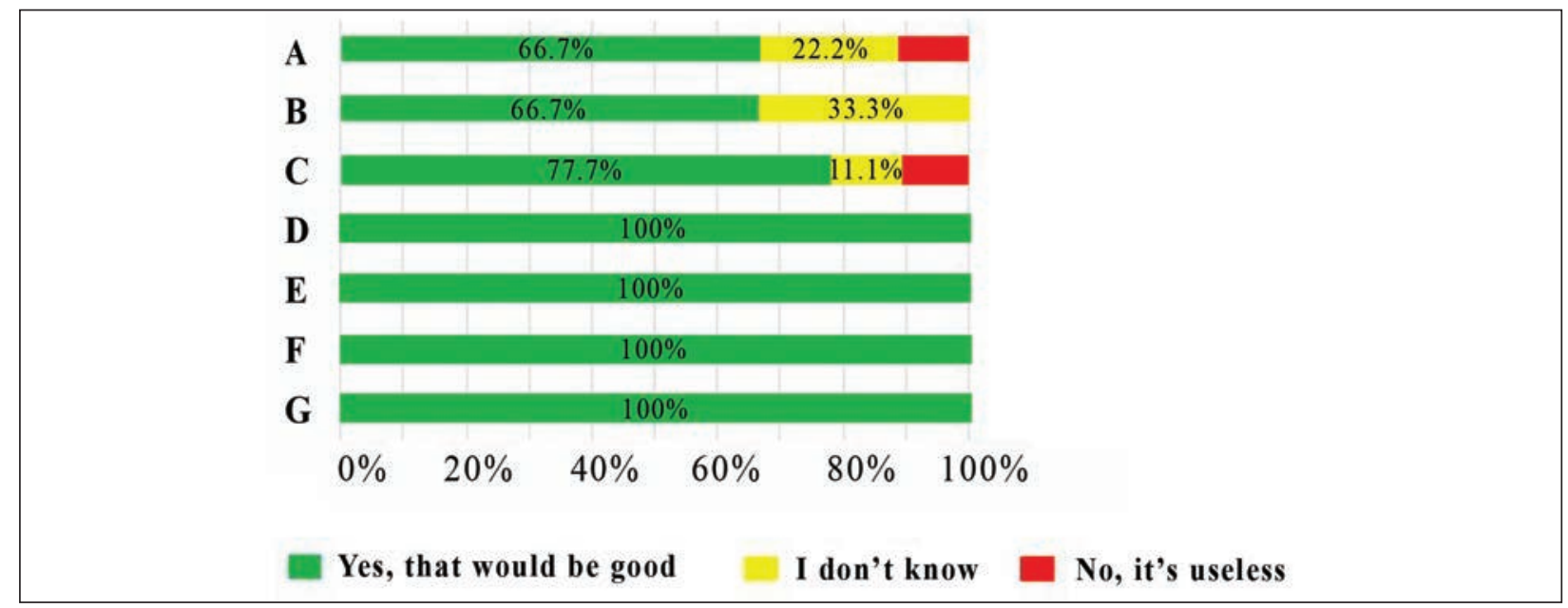

Fig. 5. Capacity enhancing features for unmanned aerial vehicles: A- Possible load weight up to $50 \mathrm{~kg}$; B - Radar equipment; C - Small arms attack function; D - Location scanning, enemy detection and marking functions; E -

High quality live streaming feature; F - Night vision equipment; $G$ - Thermal imaging equipment.

Source: authors' research result. 
Respondents agree with the features of thermal imaging, night vision and high-quality live broadcast. 100 percent of respondents agree that these features would be useful, so it can be said that there is a need for these features while performing tasks. $66.7 \%$ of the respondents agreed with the usefulness of radio localization equipment, the other $33.3 \%$ noted that they do not know whether such equipment and function is necessary.

The results showed that more than half of the respondents expressed a need for the use of radio localization equipment. The function of light weapons and attack in the tactical class UAVs is an innovative and new thing, not only in Lithuania, but also around the world. $11.1 \%$ of respondents noted that such a function is useless, $11.1 \%$ do not know and $77.7 \%$ agree and think that such a function would be useful. It is important to keep in mind that the majority of respondents answered the previous questions that one of the main functions of the UAVs is the protection of units or objects.

The logistical function in the tactical class is also a new thing and $66.7 \%$ of respondents think it would be useful, $22.2 \%$ say it would be useless and $11.1 \%$ don't know. In summary, it can be stated that for all Figure 10 . there is a need for the specified functions, as these functions would improve the quality of task performance. In addition, the need expressed by respondents is in line with the enhanced functionality UAVs parameters and it can be assumed that there is a need to use medium-altitude enhanced functionality drones for both land and air force tasks.

\subsection{Required Specifications Indicated by Respondents}

Currently, LAF SOP MPB GV has unmanned aerial vehicles that can perform only daytime reconnaissance functions, but the need expressed by the respondents is to use thermal imaging, night vision and radio location equipment. The MPB GV uses drones capable of operating within a radius of up to 10 kilometers, but the demand is up to 100 kilometers. Time in the air - MPB GV currently uses drones that can stay in the air for up to 2 hours, but the need expressed by respondents to use aircraft that can stay in the air for up to 10 hours. Lifting capacity - The UAVS currently used by MPB LUs do not have an additional cargo carrying function, but respondents have expressed the need to use an unmanned aircraft capable of carrying up to 40 kilograms. Information transmission methods currently used by LAF MPB LUs UAVS uses directional and non-directional radio communication, there is no need to change this function. After study the table of required specifications has been created (Table 1).

Table 1.

The UAVs specifications according expressed needs of LLF SOP

\begin{tabular}{|l|l|}
\hline \multicolumn{1}{|c|}{ SPECIFICATIONS } & \multicolumn{1}{c|}{ NEED EXPRESSED BY THE LLF SOP } \\
\hline Exploration Techniques & $\begin{array}{l}\text { Topographic } \\
\text { Thermal imaging } \\
\text { Night vision } \\
\text { Radio locator }\end{array}$ \\
\hline Time in the Air & $10 \mathrm{~h}$ \\
\hline Operating Radius & $100 \mathrm{~km}$ \\
\hline Information Transfer & Directional and non-directional radio communication \\
\hline Level of Autonomy & High \\
\hline Additional Features & $\begin{array}{l}\text { Location scanning } \\
\text { Target marking } \\
\text { Patrol with light weapons } \\
\text { Cargo transportation up to } 40 \text { kilograms } \\
\text { High video quality broadcasting }\end{array}$ \\
\hline
\end{tabular}

Source: authors' research result

After systematizing the respondents' answers, diagrams of available and required specifications were made. The diagrams show the difference between the available and required UAVs specifications and features. The comparative diagram was drowned. The get results are presented in the Fig. 6 . There can be seen the blow bars and the red line. The blue bars indicate the available capacity, the red line - the required capacity. 


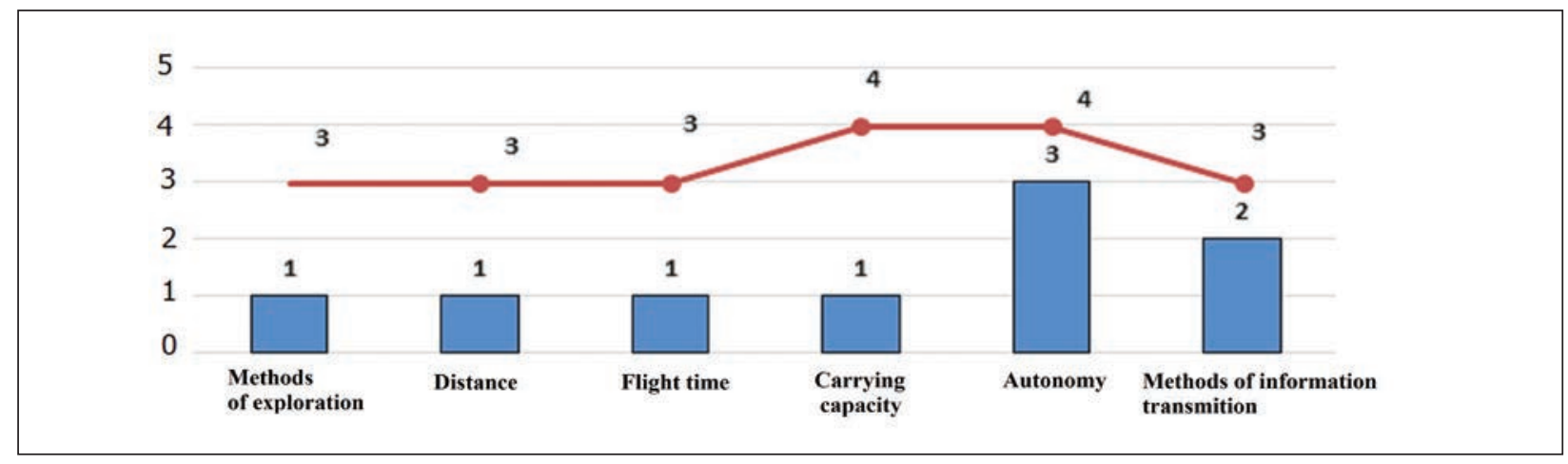

Fig. 6. The diagram of available and required specifications for UAVs according the focus group data study. Source: authors' research result

In summary, there is a significant difference between the available and required specifications and features. Currently, the MPB GV uses UAVs with daytime reconnaissance capabilities and can only reconnaissance during the day, but if it had night vision and thermal imaging equipment, it could monitor the area of operation day and night. Additional combat and logistical features would increase the combat capability and mobility of the MPB GV. In order to find out whether the desired functions would be useful, the next section examines the functionality of the UAVs of the identified needs according to Table 30 by implementing it in the main tasks performed by the MPB LV.

\section{The UAVs Capabilities Assessment}

The aim of these investigations was to evaluate the application and use of medium-altitude long endurance unmanned aerial vehicles in the Lithuanian Land Forces (LLF). According to main goal, there was carried out the analysis of the functions and tasks of the Lithuanian Armed Forces, largest brigade. The detailed results of UAVs capabilities investigation according to using them for deployment at the „Iron Wolf” brigade possibilities are presented in Table 2 and in Fig.7, Fig.8, Fig. 9 and Fig. 10.

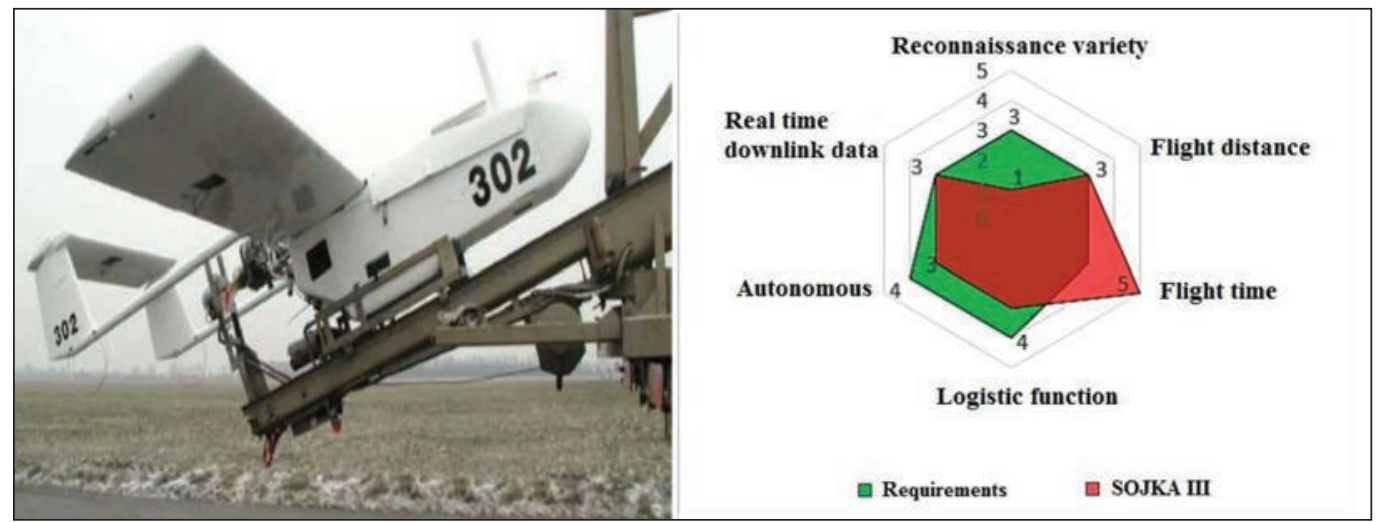

Fig. 7. The view of UAV Sojka III on launcher rails and in radar chart its capabilities assessment according to LF demands.

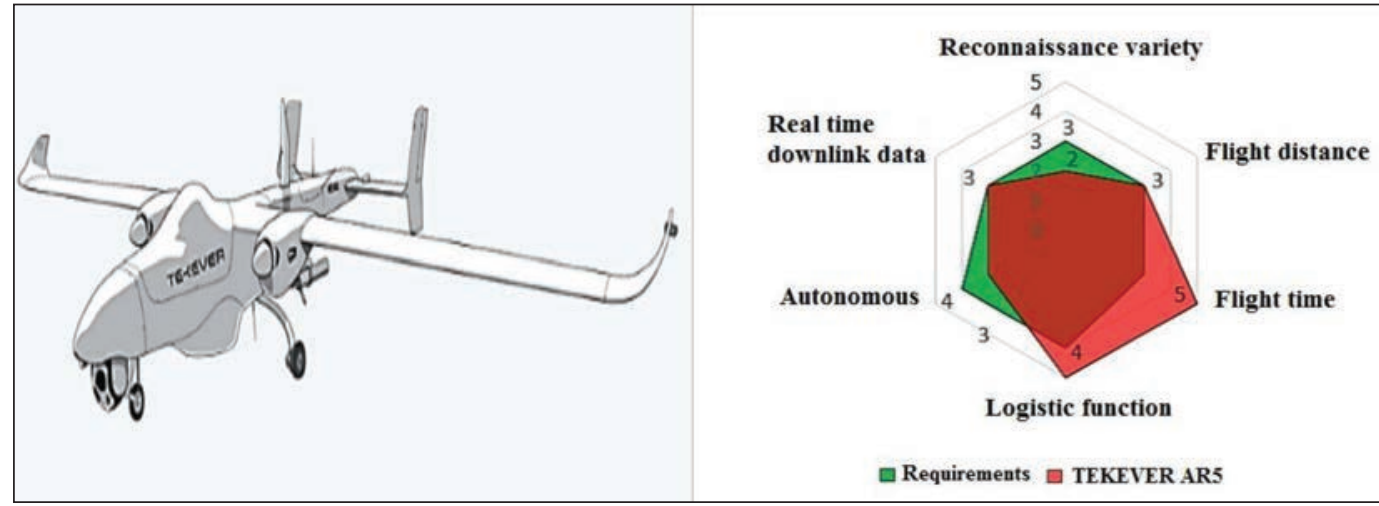

Fig. 8. The view of UAV Tekever AR5 and in radar chart its capabilities assessment according to LF demands. 


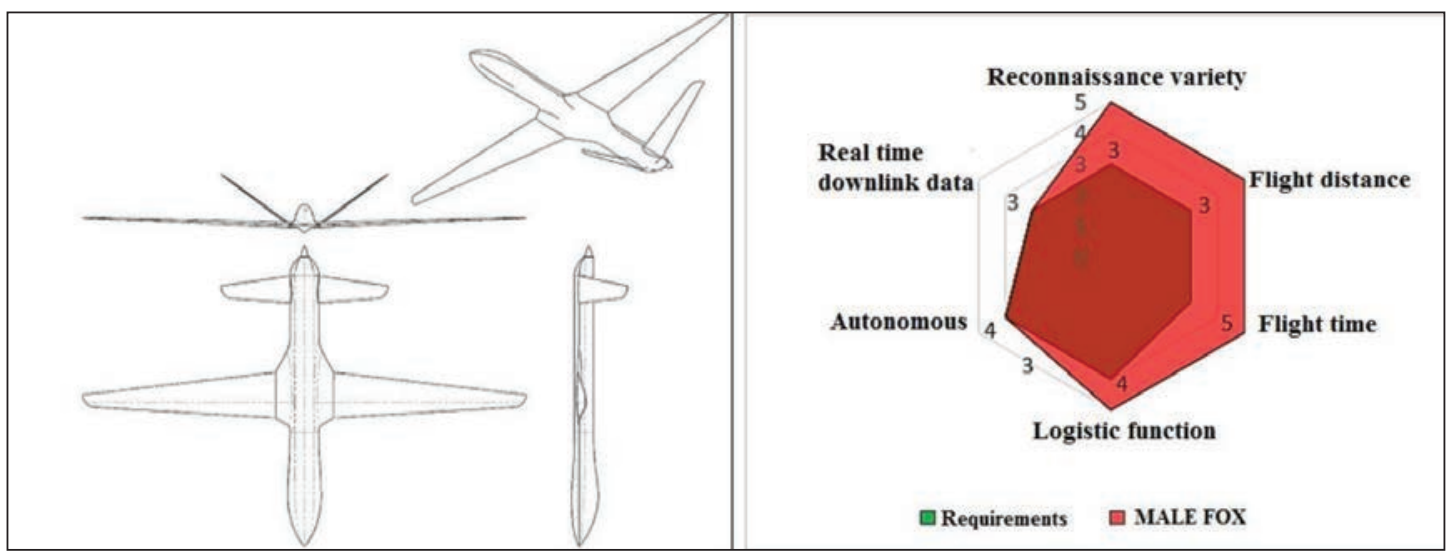

Fig. 9. The UAV Male FOX schemes under construction and in radar chart the Male FOX UAV capabilities assessment according to LF demands.

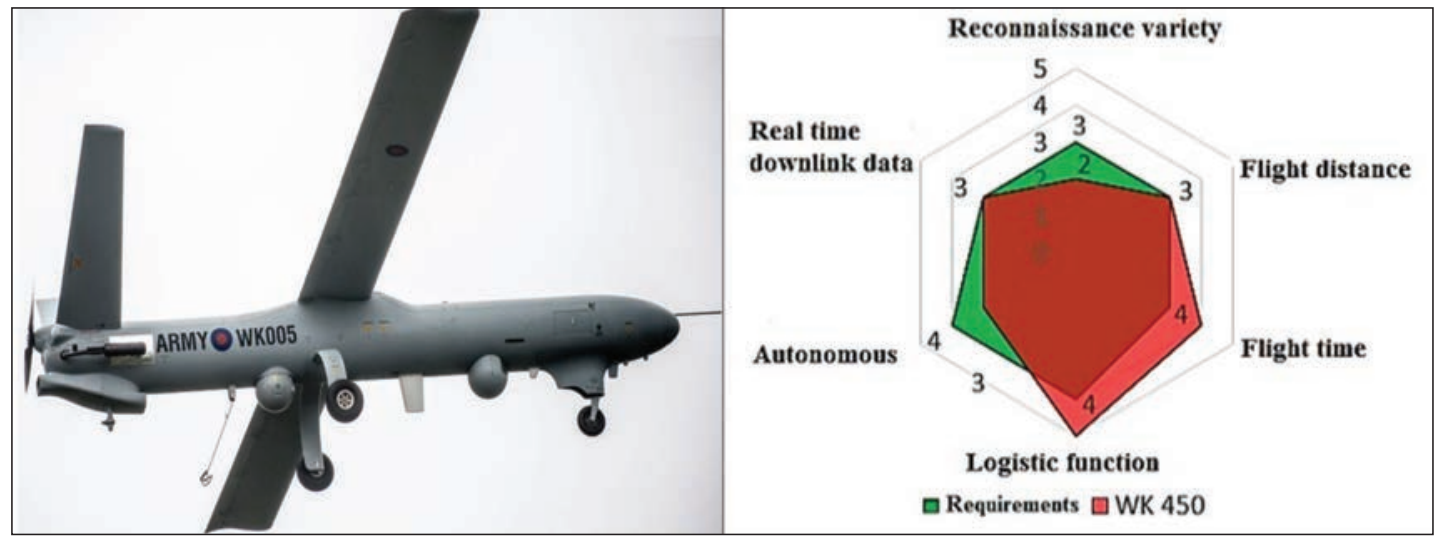

Fig. 10. The UAV Watchkeeper WK450 and in radar chart this UAV capabilities assessment according to LLF demands.

Table 2.

The UAV capabilities assessment according to LF demands

\begin{tabular}{|c|c|c|c|c|c|c|c|c|c|c|c|c|c|c|c|c|}
\hline UAVs & $\begin{array}{l}\vec{E} \\
\text { 总 }\end{array}$ & $\underset{n}{0}$ & $\frac{\overrightarrow{0}}{\frac{500}{20}}$ & 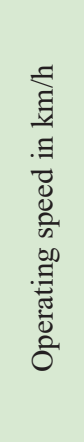 & 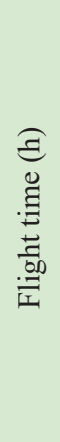 & 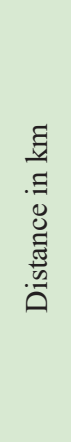 & 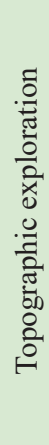 & 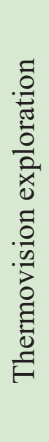 & 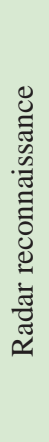 & 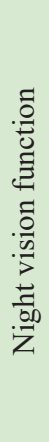 & 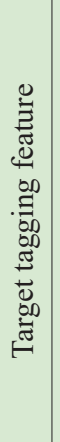 & 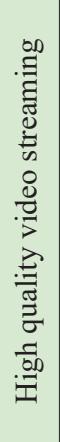 & 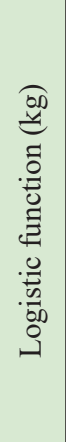 & 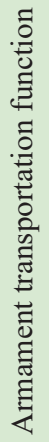 & 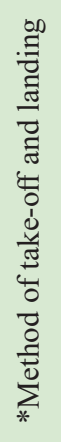 & 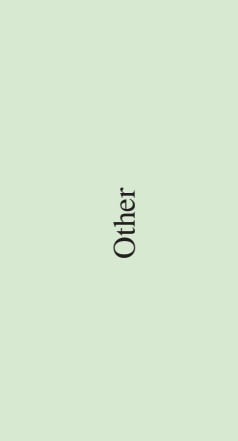 \\
\hline Requirements & - & Tactical & - & $100+$ & 10 & 100 & + & + & + & + & + & + & + & + & + & \\
\hline Sojka III & $\begin{array}{c}\text { Czech } \\
\text { Republic }\end{array}$ & Tactical & 145 & 130 & 24 & 100 & + & + & - & - & - & + & 30 & - & $1 ; 6$ & - \\
\hline Male FOX & Lithuania & Tactical & 150 & $120+$ & 24 & & + & + & + & + & + & + & 50 & + & $1-6$ & $\begin{array}{c}\text { Adjustments } \\
\text { according need }\end{array}$ \\
\hline Tekever AR5 & USA & Tactical & 180 & 100 & 20 & 100 & + & + & - & + & - & + & 50 & & $2 ; 5$ & $\begin{array}{c}\text { Auto take-off and } \\
\text { landing }\end{array}$ \\
\hline $\begin{array}{c}\text { Watchkeeper } \\
\text { WK450 }\end{array}$ & UK & Tactical & 450 & 120 & 17 & 100 & + & + & - & - & + & - & 150 & + & $2 ; 5$ & $\begin{array}{l}\text { Limited availabil- } \\
\text { ity }\end{array}$ \\
\hline
\end{tabular}

*Takeoff methods: 1 - mechanically; 2 - on the trail; 3 - by hand; Landing methods: 4 - trap; 5 - landing track; 6 - emergency landing on pillows. Source: authors' research result 
Additionally, there was conducted an analysis of the need for, and capabilities for, unmanned aerial vehicle enhanced brigade operations. The survey results let to assume that: all questioned respondents claimed that their UAV's do not have the necessary functions. According to done study, respondents' demand for basic UAV specifications are focused on: operational range $-100 \mathrm{~km} ; 10$ hours airborne; topographic, thermovision and radar reconnaissance; high quality night vision camera; $50 \mathrm{~kg}$ for combat and logistic functions. A criterion table (Table 2) has been drawn up to meet the need to select an unmanned aircraft. The Tactical Class UAVs range in size from 150 to 600 kilograms and are various, but for the best of the latest features, it was decided to judge the UAVs that were presented in the Defense \& Security Equipment International exhibition, in 2019 year, because this exhibition featured all the most innovative technologies.

Finally, this survey disclosed that available UAVs capabilities do not meet the requirements. The LAF needs to deploy advanced UAVs with special reconnaissance, cargo and combat capabilities.

The needs of LAF respondents were best met by the MALE FOX unmanned aerial vehicle, which meets all the functions indicated by LAF respondents in Table 2 and, if necessary, improvements are possible on a specific project for LASCO. After selecting MALE FOX, it is necessary to evaluate the benefits it will provide for LAF, therefore it was decided to perform a functionality assessment with all the functional possibilities of UAVs.

\section{The Benefits of MALE FOX UAV}

After reviewing and selecting the UAVs that participated in the 2019-year international defense exhibition DSEI, an unmanned MALE FOX aircraft vehicle was selected. To assess how the deployment of MALE FOX in the Land Forces would affect the capacity of the MPB LU, a functionality assessment of all MALE FOX UAVs functions is required. As a study result the MALE FOX technical parameters and possible extensions are presented in the Table 3.

Table 3.

MLE FOX UAVs technical parameters and extension capabilities description

\begin{tabular}{|l|l|}
\hline \multicolumn{2}{|c|}{ Technical parameters } \\
\hline Wing width & $850 \mathrm{~mm}$ \\
\hline Length & $9000 \mathrm{~mm}$ \\
\hline Weight & $150 \mathrm{~kg}$ MTOW \\
\hline Possible load weight & $50 \mathrm{~kg}$ \\
\hline Engine & $250-500 \mathrm{~cm} 3$ \\
\hline Maximum speed & $180-200 \mathrm{~km} / \mathrm{h}$ \\
\hline Cruising speed & $120 \mathrm{~km} / \mathrm{h}$ \\
\hline Minimum mission speed & $\begin{array}{l}90 \mathrm{~km} / \mathrm{h} \text { (when a huge amount of informa- } \\
\text { tion needs to be collected) }\end{array}$ \\
\hline Minimum Safe Speed & $55-65 \mathrm{~km} / \mathrm{h}$ (landing speed) \\
\hline Operating radius & Up to $500 \mathrm{~km}$ \\
\hline Flight time & $12 \mathrm{~h}-24 \mathrm{~h}$ \\
\hline Rise height & $9000 \mathrm{~m}$ \\
\hline
\end{tabular}

\begin{tabular}{|l|}
\hline \multicolumn{2}{|c|}{ Features of MALE FOX UAVs } \\
\hline$\checkmark$ Reconnaissance; \\
$\checkmark$ Operations; \\
$\checkmark$ For retransmission of radio or other \\
communication; \\
$\checkmark$ Logistics/ transportation; \\
$\checkmark$ Defensive / offensive \\
$\checkmark$ Target simulation \\
\hline Partnership to expand opportunities \\
\hline \\
$\checkmark$ Thermal imager manufacturers; \\
$\checkmark$ IR/NIR/S/SWIR camera \\
manufacturers; \\
$\checkmark$ Lidar producers; \\
$\checkmark$ SAR radar manufacturers; \\
$\checkmark$ Micearms suppliers. \\
\hline
\end{tabular}

Source: authors' research result

The MALE FOX unmanned aircraft can stay in the air for up to 24 hours, operate within a radius of up to $500 \mathrm{~km}$ and have high-quality live broadcast, Lidar radar system, night vision, thermal imaging and radio local reconnaissance functions. During the day or night, objects can be well-disguised and unobtrusive, but using thermal imagers and Lidar radars would make it easier and less time to spot live or accurately shaped objects.

During the battle, the headquarters could clearly see how and what tasks their units are performing. It would also see in real time when and where support units need to be sent. All these functions now are done with the help of radio procedures across all management circuits and it is time consuming. So, MALE FOX with better equipment would allow you to control units more effectively and faster during battle or exercise.

Major countries around the world like the US or Russia use combat class UAVs (Predator C and Sukhoi S-70 Okhotnik) with combat features. It is expensive to maintain such UAVs. LAF does not allocate so much funds yet, so tactical class UAVs with combat functions can be a great alternative. The MALE FOX unmanned has the ability to use smart weapons, so it can be used as an aerial support aircraft, as it has built-in combat weapons and can fire at specified or identified targets. Thus, MALE FOX unmanned aerial vehicles with combat functions could increase combat power in carrying out fire support tasks without risking the lives of soldiers, as Gheorge Udeanu argues in his 
article on combat UAVs functionality (2016).

In addition, during the movement of units in hostile territory, MALE FOX could also perform force protection tasks and increase the efficiency of their performance without risking the lives of soldiers, according to Gheorge Udeanu in his article on combat UAVs functionality . MALE FOX could fly at an altitude of several kilometers, monitoring the entire area and notifying the unit in that area when it noticed the movement of other units. After receiving information from the $\mathrm{BO}$, the battery has more time to withdraw than when receiving information from a cookie, when there is only time left to prepare for the defense, posing a risk to the lives of the soldiers.

MALE FOX can carry up to $50 \mathrm{~kg}$ of cargo, such as ammunition or food. In the case of food transport, dry rations for soldiers weighing up to 7 kilograms per box (10 units) could be transported, in which case the UAVcan lift 4 boxes (40 units) of dry rations and 22 liters of water, which is enough for a unit (up to 12 soldiers). size reconnaissance unit for the whole day and more. In the case of ammunition transport, one box of ammunition weighs 15 kilograms (1000 rounds), the UAV could lift 3 boxes of ammunition and deliver them to the unit. A soldier with a full combat kit must have 240 rounds, so MALE FOX could carry a full combat ammunition for a unit-sized unit.

With the use of such capabilities by UAVs, leadership and management tasks could be assigned in real-time by monitoring of the situation [18]. MALE FOX can be on the air for up to 24 hours and broadcast high quality video in $8 \mathrm{~K}$ resolution $(7680 \times 4320)$.

MALE FOX could also be used as a reconnaissance or search tool. The contribution of the UAV to providing assistance to other institutions or civilian agencies would be a major support in cases of search and rescue operations as was detailed discussed in Sonia Waharte study [20].

\section{Conclusions}

According to done study, respondents' demand for basic UAV specifications are focused on: operational range $-100 \mathrm{~km}$; 10 hours airborne; topographic, thermovision and radar reconnaissance; high quality night vision camera; $50 \mathrm{~kg}$ for combat and logistic functions.

The judgement of the UAVs which were presented in the Defense \& Security Equipment International exhibition, in 2019 year, disclosed that available UAVs capabilities do not meet the LAF requirements. The LAF needs to deploy advanced UAVs equipped with special reconnaissance, cargo, and combat capabilities, which were featured all the most innovative technologies.

A deep assessment of the available unmanned aerial vehicles according the required criteria for LAF UAVs let make the conclusions that the MALE FOX unmanned aerial vehicle can be the best choice for LAF because:

Only the MALE FOX unmanned aircraft meets all the criteria. The deployment of MALE FOX unmanned aircraft to the Lithuanian Armed Forces would increase combat and reconnaissance capabilities as well as reduce the risk to the lives of soldiers.

For these reasons, it was suggested to integrate the MALE FOX unmanned aircraft in the structure of the Lithuanian Armed Forces.

\section{References}

1. Ehrhard, T. P. 2010. Air force UAVs the secret history. Portland, Maine: Mitchell Institute Press.

2. Bernar, V. 2012. New technologies and warfare. Cambridge: Cambridge Journals.

3. Stansky, P. W. A. 1994. Life, death, and art in the Second World War. Stanford California: Stanford University Press.

4. Kückelhaus, D. M. 2014. Unmanned aerial vehicles in logistics. Troisdorf, Germany: DHL Customer Solutions \& Innovation.

5. László Kovács, L. V. 2015. Unmanned Aerial Vehicles in the Hungarian Defence Forces. 10th International Symposium of Hungarian Researchers on Computational Intelligence and Informatics.

6. Novaković Z. Mitrović, N. M. L. 2014. Increasing Launch Capability of a UAV Bungee Catapult. Scientific Technical Review.

7. Waharte, S. N. T. 2015. Supporting Search and Rescue Operations withUAVs. Oxford, United Kingdom: Oxford University Press.

8. Tekever AR5. 2019. [online cit.: 2019-09-28]. Available from: tekever AR5: http://airray.tekever.com/ar5/

9. Udeanu, G., Dobrescu, A., Oltean, M. 2016. Unmanned aerial vehicle in military operations.

10. Bekesiene S. 2017. Mini Unmanned Aerial Vehicle System Optimization for Lithuanian Military Demands. Transport Means 2017. Kaunas: Kaunas University of Technology, 735-740. ISSN 1822-296X.

11. IBM SPSS Statistics [online cit.: 2019-09-22]. Available from: http://www.ibm.com/analytics/us/en/technology/spss/

12. Pincevičius, A.; et al. 2008. Modeling of Infantry Attacks on Real Terrain. Nonlinear Analysis: Modelling and 
Control, vol. 13(4): 491-501. ISSN 1392-5113

13. Hošková-Mayerová Š., Talhofer V., Hofmann A., et al. 2013. Spatial Database Quality and the Potential Uncertainty Sources, Advanced Dynamic Modeling of Economic and Social Systems, Book Series: Studies in Computational Intelligence, vol. 448, 127 p.

14. Hošková-Mayerová Š., Talhofer V., Hofmann A., et al. 2013. Mathematical Model Used in Decision-Making Process with Respect to the Reliability of Geodatabase, Advanced Dynamic Modeling of Economic and Social Systems, Book Series: Studies in Computational Intelligence, vol. 448, 143 p.

15. Bekešienè, S. 2018. Protection Systems against Unmanned Aircraft Vehicle Evaluation. Bekesiene S., HoskovaMayerova S. (Eds.), Challenges to National Defence in Contemporary Geopolitical Situation (CNDCGS' 2018), 78-88, ISSN 2538-8959.

16. Čekanavičius V., Murauskas G. Statistika ir jos taikymai. 2 d. 2004, Vilnius:TEV. -270 p.

17. Austin, R. 2010. Unmanned Aircraft Systems. New Jersey: John Wiley \& Sons Ltd.

18. Guilherme L. S. V. 2015. Raspberry Drone: Unmanned Aerial Vehicle

19. Waharte, S. N. T. 2015. Supporting Search and Rescue Operations withUAVs. Oxford, United Kingdom: Oxford University Press.

20. Army technology [online cit.: 2019-09-28]. Available from: https://www.army-technology.com/projects/ watchkeeper/ 